\title{
Derivation of Rapamycin: Adventures in Natural Product Chemistry
}

\author{
Sylvain Cottens ${ }^{\text {ab }}$, Jörg Kallen, Walter Schulera, and Richard Sedranißa* \\ \$KGF-SCS Industrial Investigator Award 2017
}

\begin{abstract}
The macrolide rapamycin (1) was first described as an antifungal agent in 1975. Even though its biological target and the molecular details were yet to be discovered, rapamycin attracted our interest in the early 90s based on its reported immunosuppressive activity in transplantation models and based on findings that its mechanism of action was different from those of the known immunosuppressive agents ciclosporin and FK506. In this review we describe our efforts to chemically modify this complex and chemically very sensitive natural product. Despite the limitations regarding the reaction conditions compatible with rapamycin we discovered ways of selectively modifying specific functional groups. This allowed us, among others, to improve the stability of the parent molecule towards ring-opening. Our efforts culminated in the discovery and development of the 40-O-alkylated derivative everolimus 2 which became a useful drug in solid organ transplantation, in various cancer indications and as the active principle of the market leading drug-eluting stent.
\end{abstract}

Keywords: Everolimus · Natural products · Rapamycin

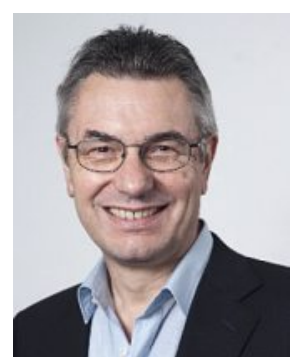

Sylvain Cottens joined Novartis in 1987 as Laboratory Head in the Nervous System Research Department. Sylvain obtained a degree in organic chemistry from the Swiss Federal Institute of Technology in Lausanne, Switzerland in 1985, and then took a postdoctoral fellowship with Prof. P. Dervan at the California Institute of Technology in the USA. From 1990 to 1994 he was a Program Head in the Immunology Department and then served as Medicinal Chemistry Unit Head in Transplantation Research. From 2000 to 2002 he was ad interim Therapeutic Area Head of Transplantation Research. Sylvain then became the Head of the Novartis Kinase and Protease Platforms (2002-2007). In 2007 he became the Global Head of the Center for Proteomic Chemistry at the Novartis Institutes for BioMedical Research (NIBR). Over the years he contributed to the discovery and development of Everolimus (Certican ${ }^{\circledR} /$ Afinitor $^{\circledR} /$ Votubia $^{\circledR}$ ) and FTY720 (Fingolimod $^{\circledR}$ ), as well as to a number of clinical candidates. After his retirement from Novartis, Sylvain joined Captor Therapeutics and works as independent consultant in the field of drug discovery.

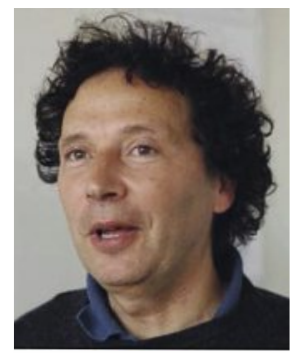

Joerg Kallen joined Novartis (at the time Sandoz AG) in 1989 after completing his PhD at the University of Basel, Switzerland (Profs. Joachim Seelig and Dieter Walz) and a postdoctoral fellowship at the Biozentrum, Basel (Prof. Johan N. Jansonius). His main specialty is X-ray crystallography and biophysics of new proteins and protein-ligand complexes. He has been working in collaboration in many disease areas (including oncology, autoimmunity, osteoporosis, transplantation, nervous system disorders, epigenetic targets), and has solved several world-new protein X-ray structures and a large variety of protein-ligand complexes (including immunophilins, nuclear receptors, protein-protein interactions, ion channels, proteases, kinases, integrins). He has contributed to the discovery of several compounds entering clinical studies.

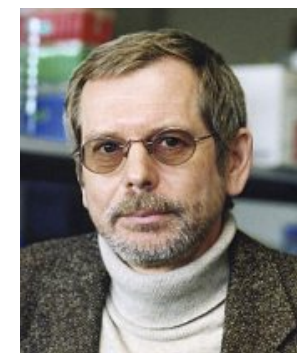

Walter Schuler holds a degree in biochemistry (University of Tübingen, Germany). $\mathrm{He}$ was trained in immunology at the University of Konstanz, Germany, from which he received a doctoral degree in 1977 (Dr. rer. nat.). His research interests focused on basic questions concerning the regulation of immune responses as well as the development of $\mathrm{T}$ and $\mathrm{B}$ lymphocytes and the molecular events involved in this process, which he pursued holding various positions in the Immunology Departments of the Universities of Konstanz and Münster (Germany), the Fox Chase Cancer Center in Philadelphia (USA) and as Member of the Basel Institute for Immunology (Basel, Switzerland). In 1991 he joined Transplantation Research Group of the Preclinical Research Department of Sandoz AG, now Novartis Pharma AG, in Basel. He was involved in various projects as Project Head in preclinical discovery and development of novel immunosuppressants. As Project Team Head he made key contributions to the discovery and pre-clinical development of everolimus. He retired from Novartis in August 2010.

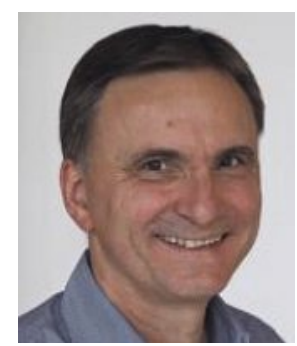

Richard Sedrani joined Novartis in 1991 after completing his $\mathrm{PhD}$ at Université Pierre et Marie Curie in Paris, France (Profs. Jean F. Normant and Alexandre Alexakis) and a postdoctoral fellowship at the University of South Carolina, Columbia, SC, USA (Prof. James A. Marshall). His drug discovery interests have been broad, ranging from natural products to many years of focus on prote- 
ase inhibition. He has been working in many disease areas, including oncology, transplantation, cardiovascular diseases, infectious diseases, ophthalmology, and has contributed to the discovery of several compounds entering clinical studies, one of which, the rapamycin derivative everolimus, has reached the market.

\section{Introduction}

Natural products and their derivatives have constituted a rich source for the treatment of diseases from ancient times through to modern pharmaceutical research. ${ }^{[1]}$ These molecules often act through mechanisms that arguably could not have been imagined by drug discoverers. Novartis has traditionally, and to this day, considered natural product research a key component of its drug discovery efforts. ${ }^{[2]}$ One of the major breakthroughs at Novartis was, for instance, the discovery of the immunosuppressive macrocyclic undecapeptide ciclosporin A which allows to prevent the rejection of allografts and which was key in enabling the practice of solid organ transplantation. ${ }^{[3]}$ The success of this drug prompted a search for alternative immunosuppressive agents and it is in this context that, in 1991, we became interested in the macrolide rapamycin (1) (Fig.1).

Rapamycin, a secondary metabolite isolated from a strain of Streptomyces hygroscopicus found in a soil sample from Easter Island, was first reported in 1975 as an antifungal agent by researchers from Ayerst. ${ }^{[4]}$ In 1989 its use as a potent immunosuppressive agent able to prevent allograft rejection in animal models was described for the first time. ${ }^{[5]}$ These experiments were prompted by the structural similarity between rapamycin and FK506 (Fig. 2), a related macrolide which had been demonstrated

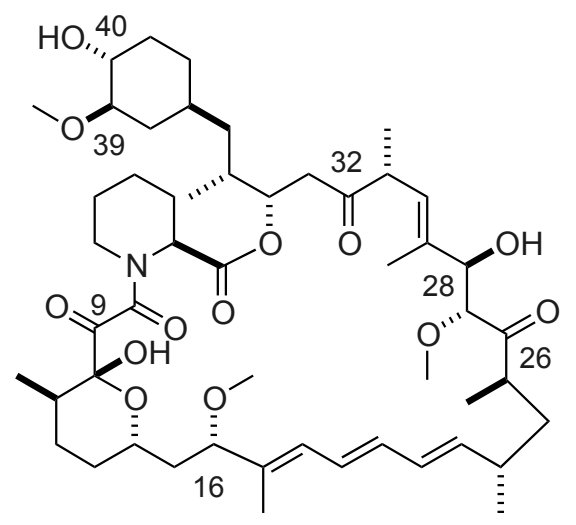

Fig. 1. Structure of rapamycin (1). to be effective in experimental transplantation and which was in development as an immunosuppressive agent at the time. ${ }^{[6]}$ Our interest was further raised by reports that, while ciclosporin and FK506, despite being very different in structure, seemed to exert their immunosuppressive activity in similar ways, the mechanism of action of rapamycin, on the other hand, appeared to be very different, although it is structurally related to FK506. Indeed it was shown that ciclosporin and FK506 prevent T-cell activation and cytokine production while rapamycin inhibits T-cell proliferation driven by cytokines like IL-2 and IL-4. ${ }^{[7]}$ Rapamycin actually inhibits growth-factor driven proliferation of many cell types, including vascular smooth muscle cells. ${ }^{[8]}$ The latter finding indicated a potential of this type of mechanism for the treatment of allograft vasculopathy leading to late graft loss. When we initiated our efforts Liu et al. reported that the cyclophilin/ciclosporin and FKBP12/FK506 complexes bind to and inhibit calcineurin. ${ }^{[9]}$ The target of the FKBP12/rapamycin complex remained unknown for a few more years until it was revealed to be the lipid kinase mTOR (Fig. 2). ${ }^{[10]}$ Even in the absence of detailed knowledge of the target at the outset of our project, the observed differences in mechanism of action between rapamycin on one hand, and ciclosporin and FK506 on the other, and the concomitant potential for combining a rapamycin derivative with ciclosporin, prompted us to embark on a chemical derivation program.

\section{Chemistry of Rapamycin}

In this review we are going to focus mainly on the chemistry challenges associated with rapamycin and on some aspects of the in vitro SAR. ${ }^{[11,12]}$ It is probably worth pointing out that this work was performed between 1991 and 1994 and is recounted here with the knowledge and the perspective of that time. When we started our work we encountered some skepticism as to whether we would be able to perform selective and efficient chemistry with this molecule at all. Indeed, as we and others quickly learned, the number and nature of the functional groups of rapamycin make it a very sensitive molecule and severely limit the range of reaction conditions which can be applied (Fig. 3).

The molecule is sensitive towards bases which can induce rearrangements in the tricarbonyl region, a retroaldol reaction and $\beta$-elimination of the pipecolinate moiety, the latter two reactions leading to ring-opened molecules. ${ }^{[13]}$ Nucleophiles in conjunction with Lewis acids result in rearrangements of the tricarbonyl region and retro-aldol reaction. ${ }^{[14]}$ Strong acids activate the allylic methoxyl, which in the worst case can lead to degradation but which can also be controlled to exchange this group as will be
Fig. 2. Cartoon illustrating that the complexes cyclophilin/ciclosporin and FKBP12/FK506 inhibit calcineurin while the complex FKBP12/ rapamycin inhibits mTOR. Inhibition of calcineurin blocks T-cell activation by downregulating the transcription of the genes for IL-2 and other cytokines. Inhibition of $\mathrm{mTOR}$ attenuates the proliferative response of lymphocytes to cytokines and of a range of other cell types to growth factors.
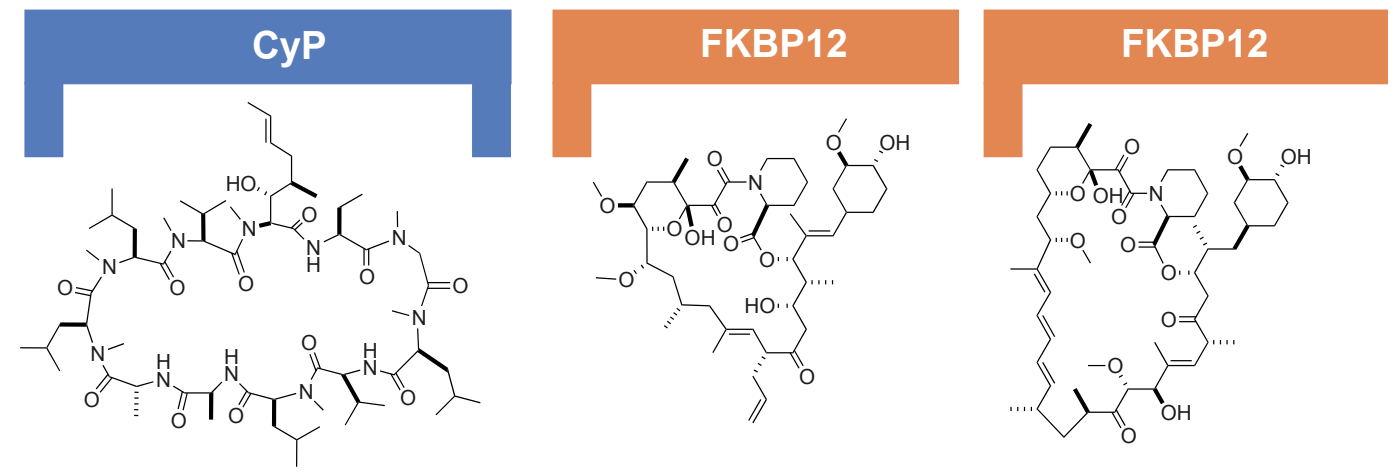

Ciclosporin A
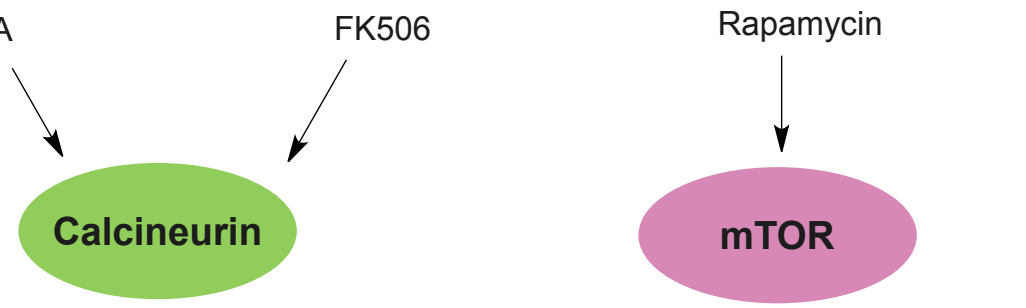


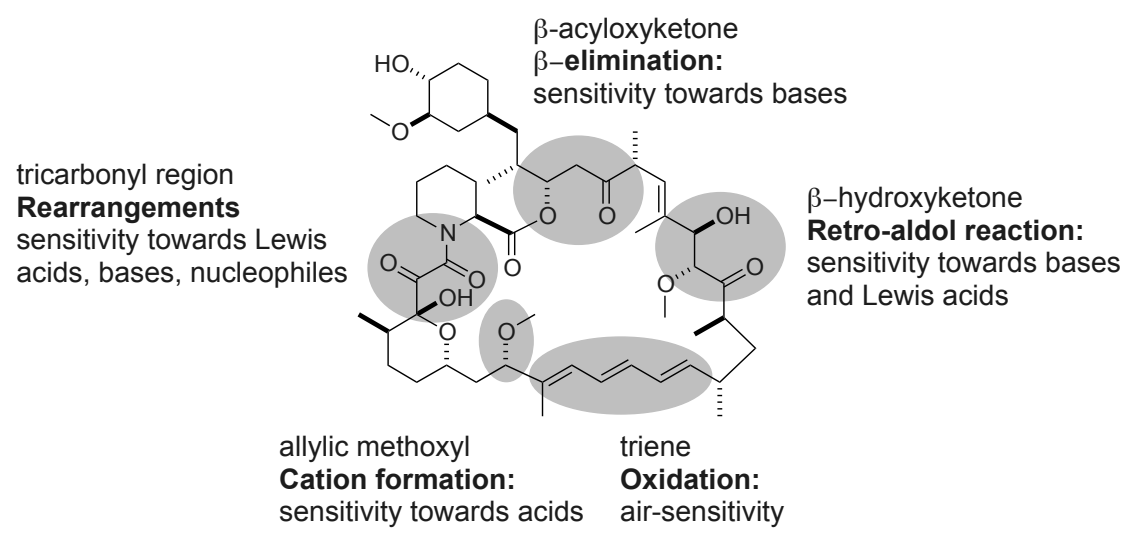

Fig. 3. Chemically sensitive

regions of rapamycin.

described below. The triene region is prone to a multitude of oxidative transformations, particularly when samples are amorphous. All of these possible side reactions make performing chemistry with rapamycin and even storing samples of derivatives a real challenge.

\subsection{Modifications of the Ketone at C(32)}

As indicated above, rapamycin readily undergoes $\beta$-elimination of the pipecolinate moiety to form a ring-opened compound which exhibits greatly reduced affinity to FKBP12 and pronounced loss of immunosuppressive activity as measured in the MLR. While we were investigating potential galenical formulations we observed that rapamycin (1) and everolimus (2) spontaneously undergo ring opening when kept in solution in a microemulsion preconcentrate at $25^{\circ} \mathrm{C}$ over 12 weeks (Scheme 1, Table 1). This degradation pathway can be attenuated by adding $2 \%$ malonic acid to the formulation.

These observations led us to search for more stable derivatives which would be less prone to $\beta$-elimination and we hypothesized that we could achieve this by modifying the carbonyl in position 32. Attempts to reduce this particular ketone to the corresponding alcohol were quite successful and we were able to achieve higher regioselectivities in comparison to efforts described in the literature around that time (Scheme 2, Table 2). ${ }^{[15]}$

Indeed, reduction of 28,40-O-bis-TES-rapamycin with 2.2 equivalents of lithium tri-tert-butoxyaluminum hydride led to reduction of both the $\mathrm{C}(9)$ and $\mathrm{C}(32)$ carbonyls, the first of which could be selectively reoxidized with $\mathrm{Cu}(\mathrm{OAc})_{2}$ to result in a 9:1 mixture of C(32) $R$ and $S$ hydroxy epimers, from which the major isomer 3 could be isolated in good yield. Reduction with lithium triethylborohydride also favored the $32 R$ epimer 3 , but the selec-

tivity was lower. Interestingly, when sodium triethylborohydride was employed, the $32 S$ epimer 4 was formed selectively and could be isolated in pure form in good yield. It is worth noting that the triethylborohydrides did not affect the C(9) carbonyl. Both $\mathbf{3}$ and $\mathbf{4}$ could be easily deprotected using HF.pyridine to give compounds 5 and 6 . We thus had quite effective means in hand to produce either of the $\mathrm{C}(32)$ hydroxy epimers of rapamycin. In their report describing the total synthesis of rapamycin Schreiber and colleagues were hypothesizing, based on analysis of the X-ray crystal structure of the FKBP12-rapamycin complex, ${ }^{[16]}$ that a secondary alcohol in C(32) with the $S$ configuration may increase the affinity for FKBP12 by acting as hydrogen bond donor in addition to the interactions formed by the hydroxyls at C(10), C(28) and C(40) of rapamycin with the $\mathrm{D} 37$ carboxylate, and the main chain carbonyls of E54 and Q53, respectively.[17] We solved the X-ray crystal structure of $\mathbf{6}$ in complex with FKBP12 and did indeed observe that the newly introduced hydroxyl makes an additional hydrogen bond with the E54 main chain carbonyl (Fig. 4), but this did not result in an increased affinity in our FKBP12 binding assay (see Table 3 below).

We also found that addition of MeLi to 28,40-O-bis-TESrapamycin results, after removal of the protecting groups, in a mixture of the two epimeric quaternary alcohols at C(32) 7 and 8 (Scheme 3). These diastereomers could be separated, but the absolute configuration of each epimer could not be assigned. Addition of trimethylsilylmethyllithium to diprotected rapamycin, followed by deprotection and concomitant Peterson olefination, led to the methylene derivative 9 in rather low yield (Scheme 4).

We were also interested in reducing the $\mathrm{C}(32)$ ketone completely down to the corresponding methylene. The usual methods, including Wolff-Kishner reductions and variants thereof, were not successful.

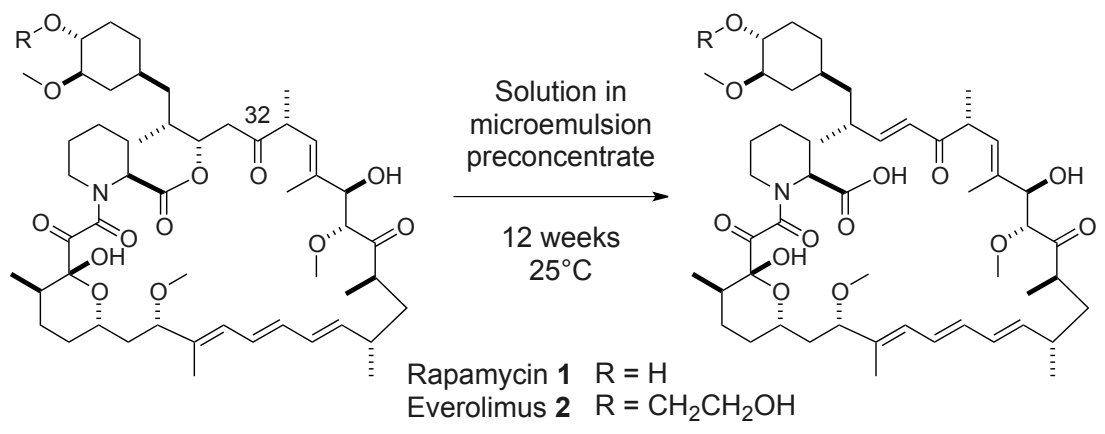

Scheme 1. Ring opening of rapamycin and everolimus in microemulsion preconcentrate.

Table 1. Compound remaining after 12 weeks at $25^{\circ} \mathrm{C}$ in solution in microemulsion preconcentrate.

\begin{tabular}{|l|c|c|}
\hline Formulation & Rapamycin [\% remaining] & Everolimus [\% remaining] \\
\hline CMPE IV & 72.7 & 73.4 \\
\hline CMPE IV, 2\% malonic acid & 97.1 & 97.3 \\
\hline
\end{tabular}



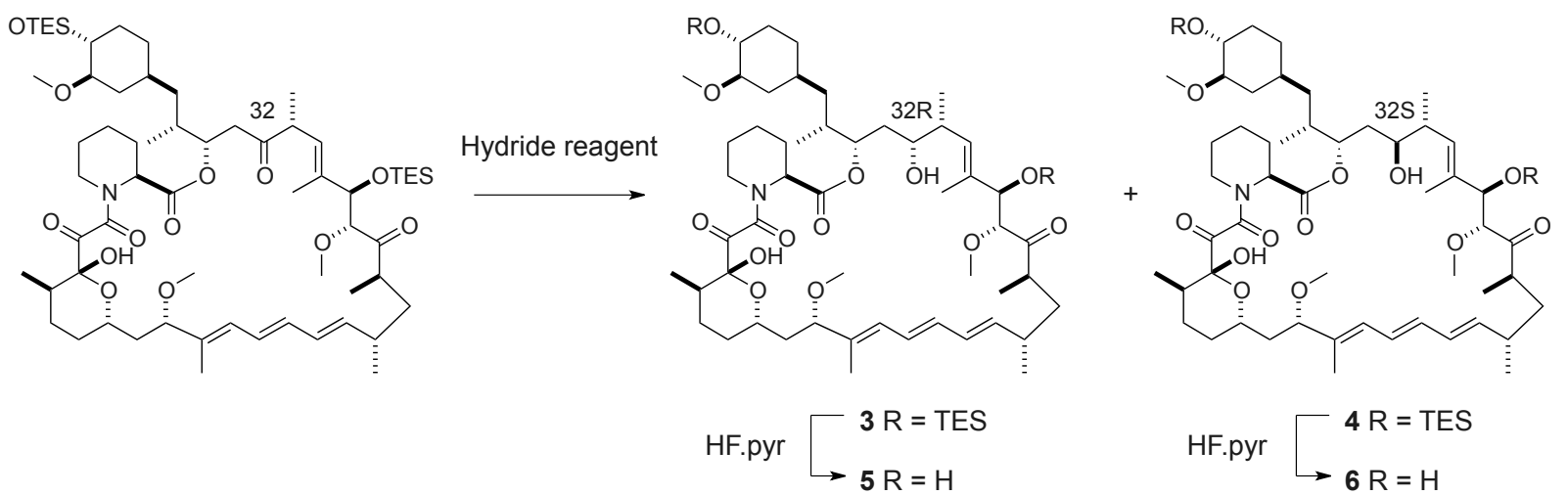

Scheme 2. Reduction of the C(32) carbonyl to the corresponding alcohol.

Table 2. Hydride reduction of the $\mathrm{C}(32)$ ketone.

\begin{tabular}{|l|l|l|l|}
\hline Reagent, equiv. & Solvent, $\mathbf{T}\left[{ }^{\circ} \mathbf{C}\right], \mathbf{t}[\mathbf{h}]$ & Yield [\%] & $\mathbf{3 2 R}: 32 S$ \\
\hline LiAlH(OtBu) $)_{3}, 2.2^{\mathrm{a}}$ & THF, -78 to 0,3 & $70^{\mathrm{b}}$ & $9: 1$ \\
\hline LiEt $_{3} \mathrm{BH}, 1.5$ & $\mathrm{THF},-78,1$ & Not determined & $3: 1$ \\
\hline $\mathrm{NaEt}_{3} \mathrm{BH}, 1.5$ & THF, -78, 1 & 72 & $1: 5$ \\
\hline
\end{tabular}

aConcomitant reduction of $\mathrm{C}(9)$-carbonyl requiring subsequent regioselectiveselective reoxidation with $\mathrm{Cu}(\mathrm{OAc})_{2}$;

boverall yield of the $32 \mathrm{R}$ epimer for the reduction-C(9) reoxidation sequence

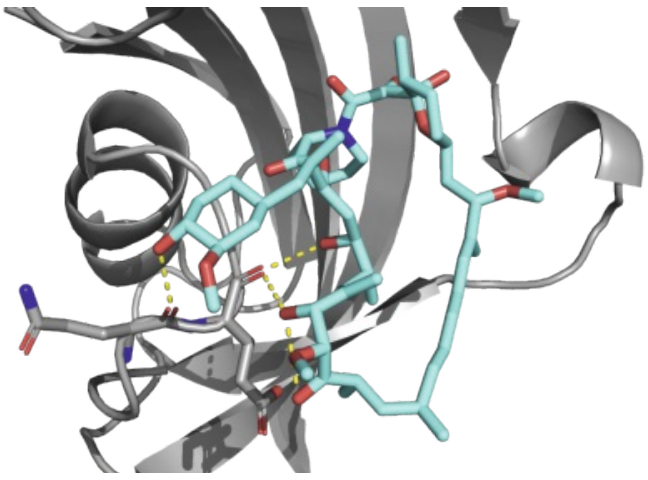

Fig. 4. X-ray crystal structure of 32S-dihydrorapamycin (6) bound to FKBP12.
The hydrazones could be prepared, but any attempt to convert them to the methylene failed. All the conditions proved to be too harsh, leading to degradation of the starting material. Consequently we had to resort to a rather lengthy route to achieve our goal (Scheme 5). [18] It started with the above mentioned reduction of 28,40- $O$-bis-TESrapamycin to the corresponding $\mathrm{C}(32) R$-alcohol 3 using lithium tri-tert-butoxyaluminum hydride followed by selective reoxidation of the concomitantly reduced C(9)-carbonyl. The C(32)-hydroxyl was transformed into the mesylate which in turn was displaced by iodide to provide compound 10. Interestingly, attempted reaction of the $\mathrm{C}(32) S$-epimer $\mathbf{4}$ with mesyl chloride failed to provide any of the corresponding mesylate. Under forcing conditions we observed largely degradation and some elimination of the C(10)-hydroxyl. Iodide $\mathbf{1 0}$ was subsequently reduced under radical conditions and
Scheme 3. Addition of MeLi to 28,40-O-bis-TES-rapamycin.

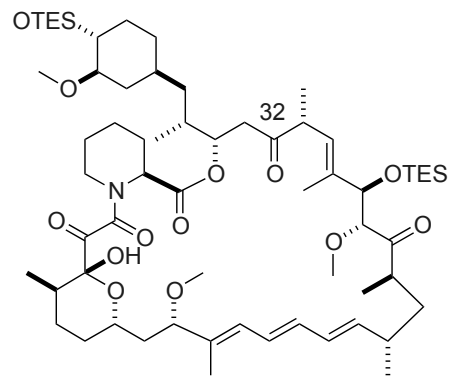

1) 2.5 eq. MeLi, $\mathrm{THF},-78^{\circ} \mathrm{C}$ 2) separate diastereomers 3) $10: 1$ HF.pyr- $\mathrm{CH}_{3} \mathrm{CN}, 0^{\circ} \mathrm{C}$

$30-40 \%$

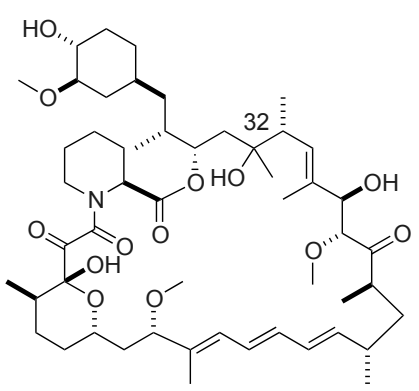

7, 8 separated epimers at $C 32$

Scheme 4. Methylenation at C(32).

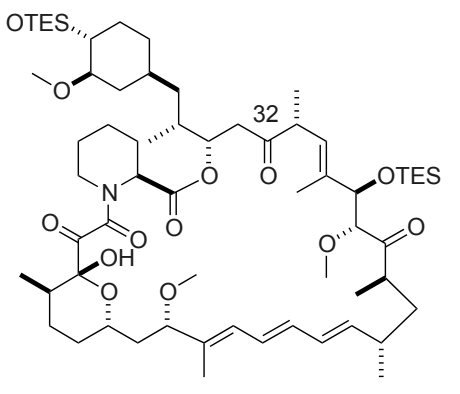

1) $\mathrm{TMSCH}_{2} \mathrm{Li}, \mathrm{THF},-78^{\circ} \mathrm{C}$ 2) $10: 1$ HF.pyr- $\mathrm{CH}_{3} \mathrm{CN}, 0^{\circ} \mathrm{C}$

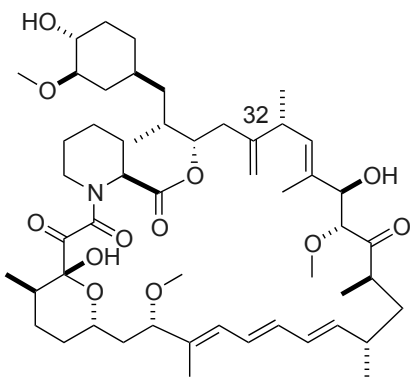




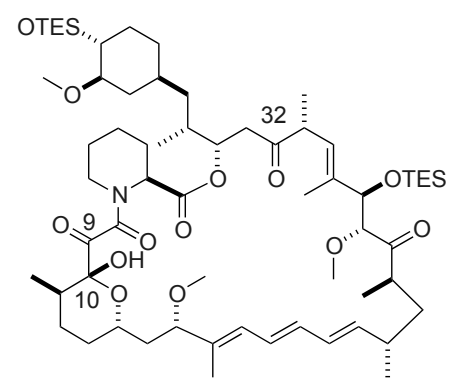

OTES

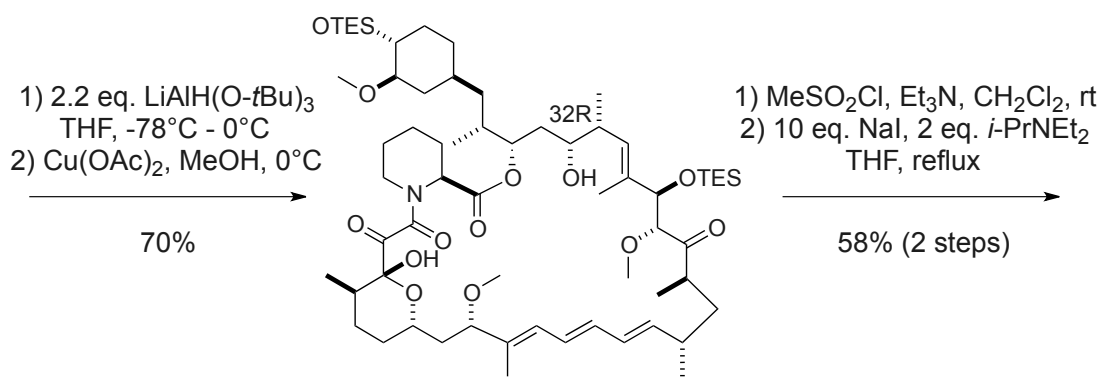

3

1) 1.5 eq. $\mathrm{Bu}_{3} \mathrm{SnH}, 15 \mathrm{~mol}_{0} \mathrm{Et}_{3} \mathrm{~B}$ toluene, rt $70 \%$

2) $\begin{gathered}1: 92 \mathrm{~N} \mathrm{H}_{2} \mathrm{SO}_{4}-\mathrm{MeOH}, 0^{\circ} \mathrm{C} \\ 61 \%\end{gathered}$

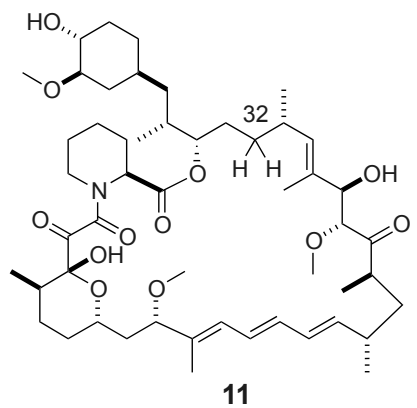

SAR 943

Scheme 5. Deoxygenation of the C(32) carbonyl.

removal of the silyl protecting groups allowed us to ultimately access the C(32)-deoxo derivative 11 (SAR943).

As can be seen from the data in Table 3 the modifications in $\mathrm{C}(32)$ did not greatly affect binding and cellular activity with the exception of compound $\mathbf{8}$ which lost some affinity and potency. As already alluded to above, compound $\mathbf{6}$ did not exhibit increased affinity to FKBP12 despite the fact that X-ray crystallography indicates an additional hydrogen bond from the newly introduced hydroxyl to the E54 main chain carbonyl. Gratifyingly the C(32)-deoxo derivative 11 showed a tendency towards increased potency in the cellular assays compared to rapamycin. A critical question was whether we had indeed increased the stability of our derivatives towards the ring-opening reaction. These derivatives were stable when treated with DBU, conditions which rapidly lead to $\beta$-elimination of the pipecolinate in the case of rapamycin. There was also interest in determining whether there was any change in stability in biological fluids. Access to bioanalytics was essentially non-existent for us, so we had to resort to an indirect way of assessing the stability. This was done by incubating rapamycin and derivatives in rat serum and determining FKBP12 binding after different incubation times. The thinking was that, for compounds undergoing ring opening and thereby losing affinity to FKBP12, the concentration of intact compound would decrease over time and we therefore should observe a shift of the inhibition curves towards higher nominal concentrations needed to achieve the same degree of binding.

The left plot in Fig. 5 shows the data obtained for rapamycin. There is indeed a shift of the inhibition curves towards the right after increasing incubation times indicating gradual ring-

Table 3. SAR of C(32)-modified rapamycin derivatives. ${ }^{[11]}$

\begin{tabular}{|l|l|l|l|}
\hline Compound & FKBP12 $\left(\mathbf{r I C}_{\mathbf{5 0}}\right)$ & IL6-dep.prol. $\left(\mathbf{r I C}_{\mathbf{5 0}}\right)$ & MLR $\left(\mathbf{r I C}_{\mathbf{5 0}}\right)$ \\
\hline $\mathbf{5}$ & 2.5 & 3.5 & 2.3 \\
\hline $\mathbf{6}$ & 1.1 & 1.1 & 0.7 \\
\hline $\mathbf{7}$ & 3.4 & 1.1 & 0.7 \\
\hline $\mathbf{8}$ & 12.0 & 10.8 & 6.6 \\
\hline $\mathbf{9}$ & 2.5 & 0.8 & 1.0 \\
\hline $\mathbf{1 1}$ & 1.2 & 0.4 & 0.4 \\
\hline
\end{tabular}
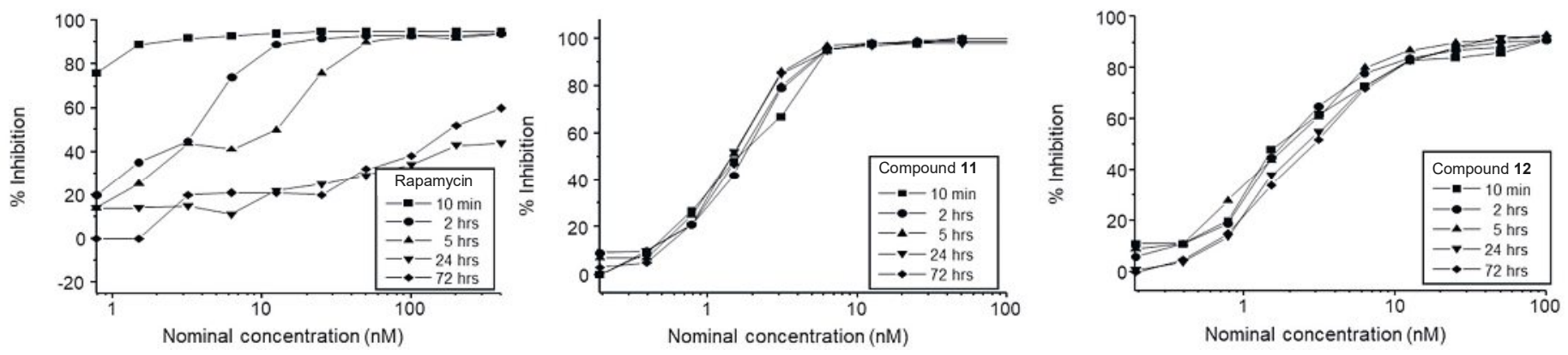

Fig. 5. Determination of the stability of rapamycin derivatives in rat serum using the FKBP12 binding assay. 


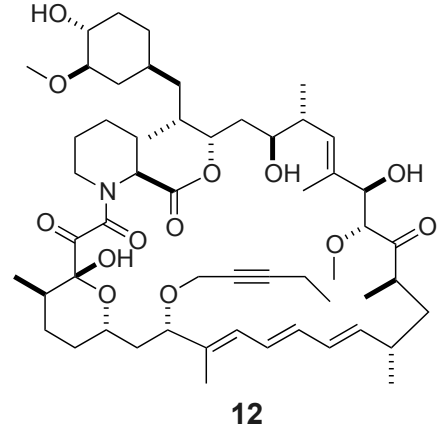

Fig. 6. Structure of compound 12.

opening and decrease of material able to bind to FKBP12. The curves for the C32-deoxo derivative $\mathbf{1 1}$ [and also for compound 12 (Fig. 6), a C(32)S-hydroxy derivative, see section 2.2, below), are essentially identical over a 72 hour period, indicating that these compounds are stable towards $\beta$-elimination in rat plasma.

\subsection{Exchange of the Methoxy Group at C(16)}

In the course of our chemistry explorations we were wondering whether it would be possible to oxidize the $\mathrm{C}(17)$-methyl group and thus gain a handle for further modifications. Treatment of rapamycin with selenium dioxide in ethanol did lead to a new product, but instead of the expected (or hoped for) allylic alcohol $\mathbf{1 3}$ we isolated compound $\mathbf{1 4}$ where the methoxy group in position 16 had been exchanged for an ethoxy (Scheme 6).

We hypothesized that selenous acid monoethylester formed in the reaction mixture was protonating the $\mathrm{C}(16)$-methoxyl, creating a cation or at least a partial positive charge, which was then intercepted by ethanol. This unexpected finding led us to wonder whether it would be possible to use this chemistry to explore a wider range of alkoxy substituents in $\mathrm{C}(16)$. As it turned out, treatment of rapamycin with an excess of an alcohol in the presence of pTsOH or TFA did indeed lead to exchange of the methoxy group in a controlled fashion (Scheme 7). ${ }^{[19]}$ The reaction proved to be applicable to a wide range of alcohols. Conversions were generally higher than $90 \%$. Usually $\sim 5: 1$ mixtures of the desired $16 S$ and $16 R$ epimers were obtained and separated by preparative RP HPLC. None of the potential regioisomers resulting from a shift of one or several double bonds was observed.

Exchange of the methoxy group did not greatly affect binding to FKBP12 (Table 4). On the other hand, introduction of ethoxy and benzyloxy moieties led to some loss of activity in the cellular assays, and this was even more pronounced when the bulkier isopropoxy group was introduced. Interestingly, introduction of propargyloxy substituents led to a 3-fold increase in activity. Compound $\mathbf{1 2}$ (Fig. 6), which is doubly modified with a pent-2-ynyloxy group in C(16) and a C(32)S-hydroxyl, exhibited an even higher increase of activity in the MLR while being stable in rat serum (section 2.1, above). Thus, the fortuitous discovery of the acid-promoted alkoxy exchange reaction in $\mathrm{C}(16)$ resulting from failed allylic oxidation attempts provided us a means of accessing derivatives exhibiting increased potency invitro. It was shown by others that $\mathrm{C}-\mathrm{C}$ bonds can be formed in $\mathrm{C}(16)$ under Friedel-Crafts type conditions using heterocycles as nucleophiles in the presence of protic or Lewis acids. ${ }^{[20]}$

\subsection{O-Alkylations}

We became interested in exploring $O$-alkylations as a potential means of introducing a range of substituents and functionalities in
Scheme 6. Attempted allylic oxidation.
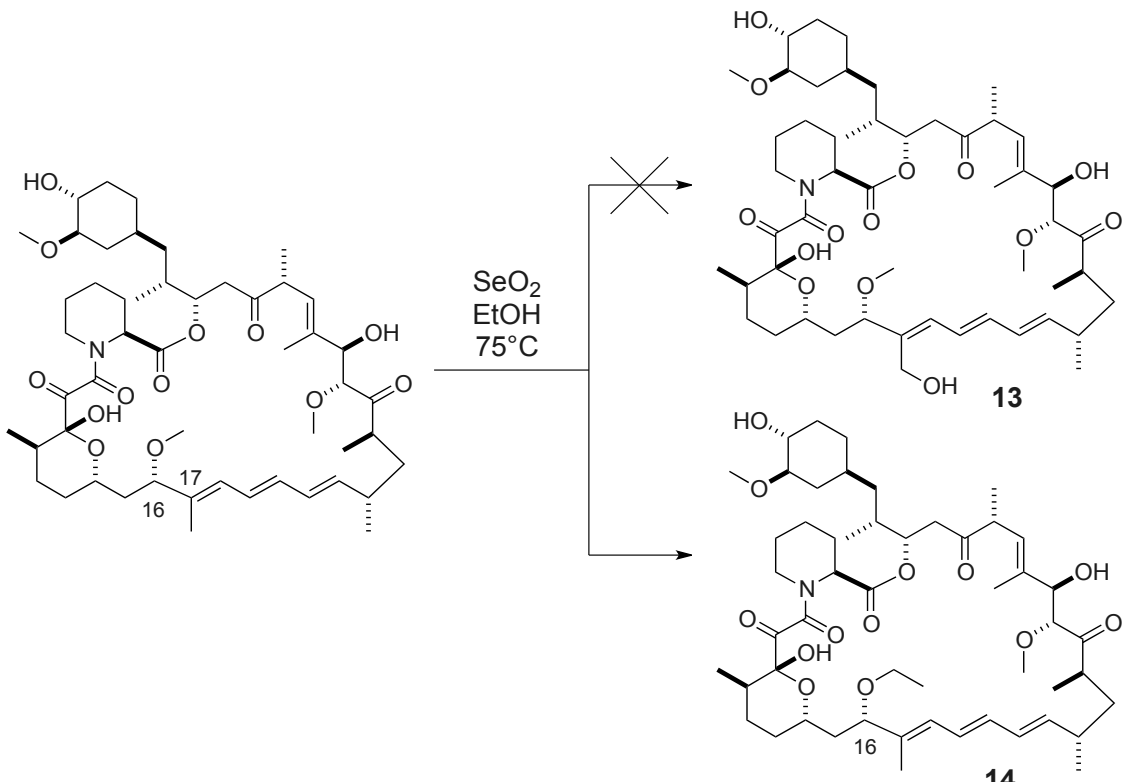

Scheme 7. Ether exchange in position 16.

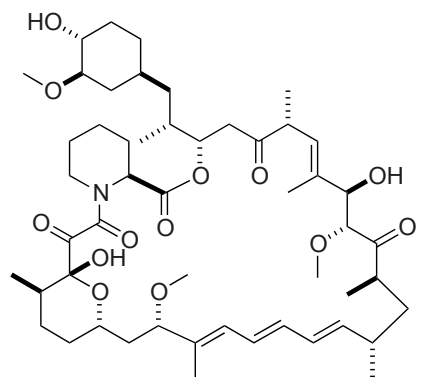

10-20 eq. $\mathrm{ROH}$ $10-20 \mathrm{~mol} \% \mathrm{pTsOH}$ $\mathrm{CH}_{2} \mathrm{Cl}_{2}$, rt, 4-8h or

3-6 eq. TFA $\mathrm{CH}_{2} \mathrm{Cl}_{2}, 0^{\circ} \mathrm{C}, 3-6 \mathrm{~h}$ 
Table 4. SAR of C(16)-modified rapamycin derivatives. ${ }^{[11]}$

\begin{tabular}{l|l|l|l|}
\hline R- & FKBP12 $\left(\mathbf{r I C}_{\mathbf{5 0}}\right)$ & IL6-dep.prol. $(\mathbf{r I C}$ & MLR $\left(\mathbf{r I C} \mathbf{C}_{\mathbf{5 0}}\right)$ \\
\hline $\mathrm{CH}_{3} \mathrm{CH}_{2}{ }^{-}$ & 1.1 & 30.0 & 12.0 \\
\hline$\left(\mathrm{CH}_{3}\right)_{2} \mathrm{CH}-$ & 0.6 & 212 & n.d. \\
\hline $\mathrm{PhCH}_{2}-$ & 0.8 & 1.1 & 12.9 \\
\hline $\mathrm{CH}_{3} \mathrm{C} \equiv \mathrm{CCH}_{2}-$ & 0.6 & 0.3 & 0.3 \\
\hline $\mathrm{CH}_{2} \mathrm{CH}_{3} \equiv \mathrm{CCH}_{2}-$ & 0.7 & 0.3 & 0.3 \\
\hline $\mathbf{1 2}$ & 1.6 & n.d. & 0.1 \\
\hline
\end{tabular}

the hope that this would allow to modulate the physicochemical properties of the parent molecule and improve our ability to formulate the active compounds. Several questions had to be addressed. In view of the sensitivity of rapamycin it was unclear whether we would find conditions mild enough to achieve $O$-alkylation. The parent molecule contains three hydroxy groups at positions 10 , 28 and 40, potentially raising a regioselectivity challenge. Finally, all of these hydroxyls are engaged as hydrogen bond donors in interactions with FKBP12. $O$-alkylation of any of the hydroxyls would be expected to disrupt the respective hydrogen bond. It was unclear whether this would negatively affect binding to FKBP12, a prerequisite for immunosuppressive activity. There was also the risk that introduction of $O$-alkyl groups would interfere with binding of the FKBP12-rapamycin complex to its target mTOR, which, as mentioned above, was unknown at the time when this work was performed.

Our efforts started with $O$-methylation attempts. As shown in Fig. 7 and Table 5 this proved to be a real challenge. Under a variety of basic conditions, including reputedly mild ones, we observed either decomposition or $\beta$-elimination of the pipecolinate followed by methylation of the resulting carboxylate to form methyl ester $\mathbf{1 5}$. When thallium ethoxide was used as a base we observed $\beta$-elimination and retroaldol reaction resulting in the isolation of the subunit 16. Finally we succeeded in performing $O$-methylation using methyl triflate. The reaction was quite selective, as 40-O-methylrapamycin (17) was obtained in $60 \%$ yield along with $13 \%$ of 28,40 -bis- $O$-methylated material.

Encouraged by this result we proceeded to alkyltriflates bearing functional groups. This chemistry is illustrated in Scheme 8 by the synthesis of everolimus (2). ${ }^{21]}$

$O$-alkylation of rapamycin with the TBDMS-protected triflate of ethylene glycol 18 proceeded quite efficiently and selectively to afford $\mathbf{2}$ after removal of the protecting group. While optimizing the reaction conditions we found that Hünig's base was more effective than 2,6-di-t-Bu-4-Me-pyridine, a base more commonly used with alkyl triflates, or 2,6-lutidine. The triflate $\mathbf{1 8}$ could be prepared separately and isolated or could be used in situ by addition of rapamycin directly to the preformed alkylating agent. This chemistry enabled the introduction of a range of functionalized alkyl groups. ${ }^{[21]}$ Nevertheless we also encountered limitations (Scheme 9). Indeed, depending on the chain length of the TBDMSO-alkyl triflate we observed formation of 40-O-TBDMSrapamycin (19) instead of the expected $O$-alkylation product. Presumably, in cases where the formation of 5- or 6-membered

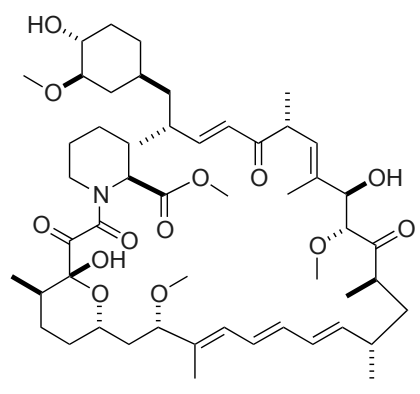

15

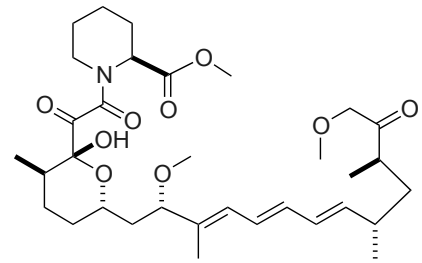

16

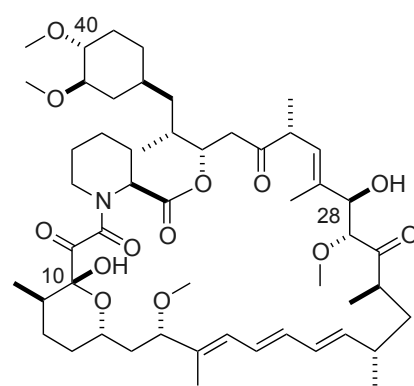

17

Fig. 7. O-Methylation attempts.

Table 5. O-Methylation attempts.

\begin{tabular}{|c|c|c|}
\hline Entry & Reaction Conditions & Result \\
\hline 1 & $\mathrm{MeI}, \mathrm{NaH}, \mathrm{DMF}, 0{ }^{\circ} \mathrm{C}$ & Decomposition \\
\hline 2 & $\mathrm{MeI}, t$-BuOK, THF, $0{ }^{\circ} \mathrm{C}$ & $\mathrm{X}+$ rapamycin \\
\hline 3 & $\mathrm{MeI}, \mathrm{Ag}_{2} \mathrm{O}, \mathrm{DMF}, \mathrm{rt}$ & $\mathrm{X}+$ rapamycin \\
\hline 4 & MeI, TlOEt, $\mathrm{CH}_{3} \mathrm{CN}, \mathrm{rt}$ & $26 \% \mathrm{Y}+$ decomposition \\
\hline 5 & $\mathrm{MeI}, \mathrm{Cs}_{2} \mathrm{CO}_{3}, 18$-crown-6, $\mathrm{CH}_{3} \mathrm{CN}, \mathrm{rt}$ & $\mathrm{X}$ \\
\hline 6 & $\mathrm{MeI}, 40 \% \mathrm{NaOH}$, cat. $\mathrm{Bu}_{4} \mathrm{NHSO}_{4}, \mathrm{CH}_{2} \mathrm{Cl}_{2}, \mathrm{rt}$ & Decomposition \\
\hline 7 & MeOTf, 2,6-di- $t$ Bu-pyridine, toluene, rt & $\begin{array}{l}60 \% \text { 40-O-Me-rapamycin } \\
\text { (+ } 13 \% \text { 28,40-bis-O-Me-rapamycin) }\end{array}$ \\
\hline
\end{tabular}


Scheme 8. Selective 40-O-alkylation exemplified by the synthesis of everolimus.

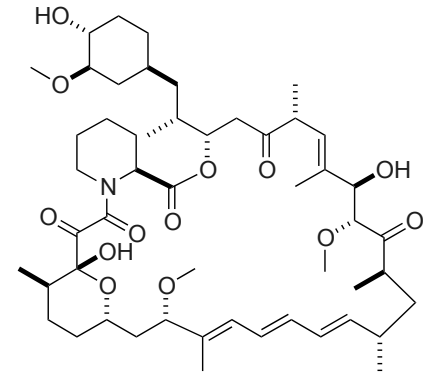

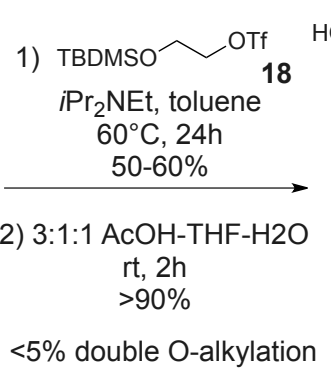

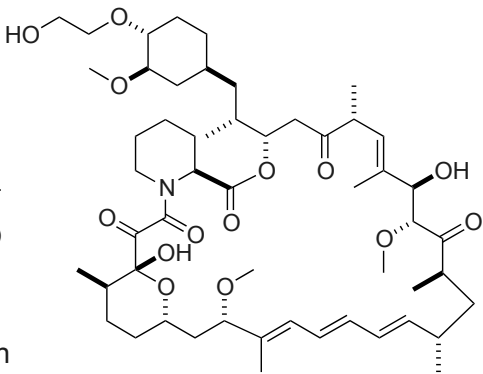

2

Everolimus (SDZ RAD, RAD001)
Scheme 9. Limitations of $O$-alkylations using functionalized alkyl triflates.

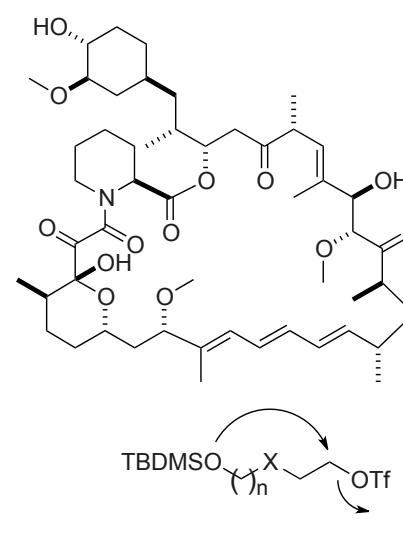

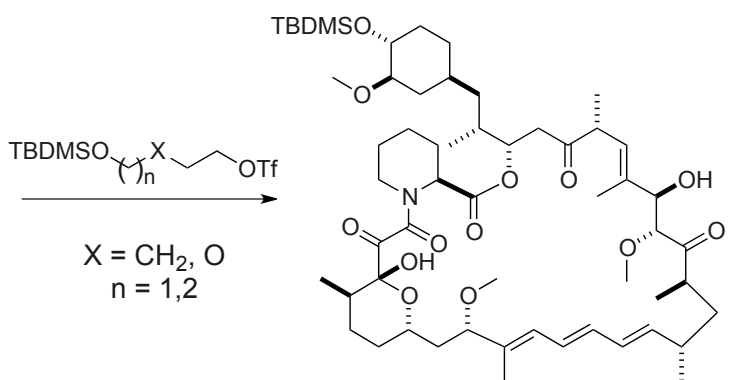

19

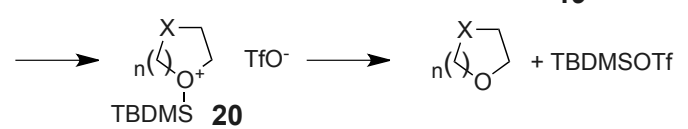

cyclic ethers is possible, the silylether oxygen displaces the triflate in an intramolecular fashion, leading to 20. This intermediate can then either serve as a silylating agent itself or generate TBDMSOTf.

The in vitro activities of some 40- $O$-alkylated derivatives are shown in Table 6. ${ }^{[22]}$ Interestingly, the affinity to FKBP12 is hardly affected despite the loss of a hydrogen bond to the Q53 main chain carbonyl, except when a bulkier substituent like phenyl is introduced. Methylation leading to $\mathbf{1 7}$ results in a 6-fold decrease of activity, while introduction of the 2-hydroxyethyl side chain leads to everolimus (2) with an immunosuppressive activity comparable to that of rapamycin. Extension to the longer 6-hydroxyhexyl chain results in a notable almost 20 -fold loss of activity.

We generated a X-ray crystal structure of everolimus (2) bound to FKBP (Fig. 8). The overlay of rapamycin and everolimus bound to FKBP12 (Fig. 8a) shows that the two compounds almost perfectly overlap. The loss of the hydrogen bond to the Q53 main chain carbonyl results in a slight upward shift of the cyclohexyl subunit which, as mentioned above, is of almost no consequence for the FKBP12 affinity and the immunosuppressive activity. In 1996, after our rapamycin project was terminated, the X-ray structure of the FKBP12-rapamycin-FRB (FKBP12-rapamycin-binding domain of mTOR) was published. ${ }^{[23]}$ The overlay of FKBP12-bound everolimus with this ternary complex (Fig. 8b) shows that neither the newly introduced side chain nor the slightly different orientation of the cyclohexyl moiety are expected to interfere with ternary

Table 6. In vitro activities of $40-0$-alkylated rapamycin derivatives. ${ }^{[11]}$

\begin{tabular}{|l|l|l|}
\hline O-alkyl group & FKBP12 $\left(\mathbf{r I C}_{\mathbf{5 0}}\right)$ & MLR $\left(\mathbf{r I C} \mathbf{5 0}^{\mathbf{}}\right)$ \\
\hline $\mathrm{Me} \mathbf{1 5}$ & 1.1 & 6.5 \\
\hline $\mathrm{HO}\left(\mathrm{CH}_{2}\right)_{2}-\mathbf{1 7}$ & 2.0 & 2.1 \\
\hline $\mathrm{HO}\left(\mathrm{CH}_{2}\right)_{6}-$ & 0.8 & 18 \\
\hline $\mathrm{Ph}-$ & 23 & $>430$ \\
\hline
\end{tabular}

complex formation, thereby providing a post hoc rationale for the retained immunosuppressive activity.

In 1996 we published the X-ray structure of 28-O-methylrapamycin 21 (Fig. 9) bound to FKBP12.[24]

This compound exhibits similar affinity to FKBP12 than rapamycin, but its immunosuppressive activity is reduced more than 1000-fold. We noticed that the macrocyclic part of $\mathbf{2 1}$ bound to FKBP12 is superimposable with the corresponding part of rapamycin (Fig. 10a). On the other hand the newly introduced methyl group pushes the cyclohexyl group away. The conformation adopted now by the cyclohexylethyl subunit is reminiscent of the orientation of the cyclohexylethenyl moiety of FK506 bound to FKBP12. The addition of the methyl group to the $\mathrm{C}(28)$ hydroxyl and the concomitant movement of the cyclohexyl result in the loss of two hydrogen bonds without major consequence for affinity to FKBP12. In our 1996 paper we hypothesized that the cyclohexyl subunit in its new position might sterically interfere with binding to mTOR. A few days after our article appeared the above-mentioned X-ray structure of the FKBP12-rapamycinFRB complex was reported.[23] The overlay of FKBP12-bound 28-O-methylrapamycin (21) with this ternary complex clearly shows that the cyclohexyl in its new position would bump into the C-terminal end of the $\alpha 1$ helix of FRB (Fig. 10b). The new conformation adopted by part of the ligand is not compatible with ternary complex formation thereby explaining the loss of activity.

\subsection{Everolimus}

Among the derivatives synthesized and profiled in the course of our rapamycin derivation program everolimus (2) was selected for further development. The compound entered $\mathrm{PhI}$ clinical trials in 1997 and was developed in parallel in kidney and heart transplantation. It received EU approval for these indications in 2003 (Certican ${ }^{\circledR}$ ) and was later approved by the FDA for kidney transplantation (Zortress ${ }^{\circledR}$ ). In the early 2000s Novartis also started developing the drug in different types of cancers. Everolimus was approved under the tradename Afinitor ${ }^{\circledR}$ for kidney cancer (2009), for the treatment of benign brain tumors associated with tuberous sclerosis complex (2010) as well as for non-cancerous 

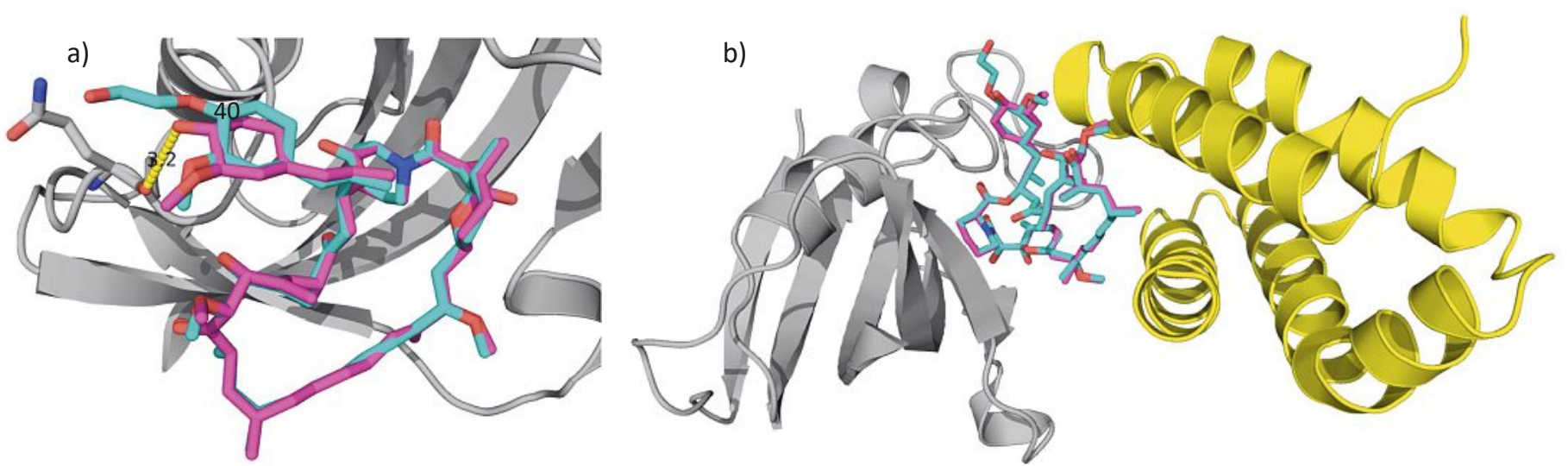

Fig. 8. a) Overlay of rapamycin (magenta) and everolimus (2, cyan) bound to FKBP12. b) Overlay of FKBP12-bound everolimus (2, cyan) with the FKBP (grey) - rapamycin (magenta) - FRB (yellow) X-ray structure. ${ }^{[23]}$

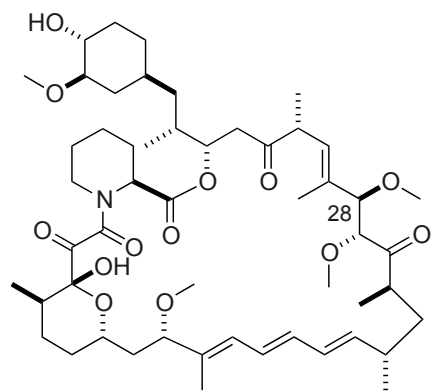

21

FKBP12 $\mathrm{rIC}_{50} \quad 1.6$ ${\text { MLR } \mathrm{rIC}_{50}} 1300$

Fig. 9. 28-O-Methylrapamycin.

kidney tumors associated with TSC (2012), for the treatment of pancreatic neuroendocrine tumors (2011) and for advanced breast cancer (2012). In addition, everolimus is the active principle in the market-leading Xience ${ }^{\circledR}$ drug-eluting stent from Abbott.

\section{Conclusion}

In this article we have reviewed aspects of the chemical derivation of rapamycin we performed between the early and mid nineties. Even if this dates back a while, we hope we could share some of the excitement of working with this molecule, both from the chemical and biological perspective. It continues to fascinate researchers and drug discoverers even today, as various research groups and companies continue to search for novel rapamycin analogs. This macrolide presents many challenges to the organic chemist and, as mentioned above, its chemical sensitivity severely restricts the range of reactions that can be performed. We used to jokingly say that this is really a case of the molecule deciding about the chemistry and not the chemist. Nevertheless, we were able to discover ways of selectively manipulating functional groups of rapamycin, sometimes even quite efficiently. Fortunately, and sometimes surprisingly, some of the modifications resulted in compounds for which the activity could be preserved. Finally, we were able to come up with a few drug candidates, one of which, everolimus, was developed. It eventually became a useful drug in transplantation, in various cancer indications and as the active principle of the market-leading drug eluting stent.

\section{Acknowledgements}

We would like to thank all people at Novartis $R \& D$ involved in the rapamycin project, and in particular: Hans Fliri, Bradley Herberich, Hans Hofmann, Lyn H Jones, Heinz Jundt, Anne-Marie Jutzi Spony, Klaus Memmert, François Nuninger, Dieter Oser, Max Schreier, HenkJan Schuurman, Binh Thai, Gerhard Zenke, Mauro Zurini.

Received: May 28, 2019

[1] R. Bade, H.-F. Chan, J. Reynisson, Eur. J. Med. Chem. 2010, 45, 5646, doi: 10.106/j.ejmech.2010.09.018.

[2] a) A. Hofmann, Pharmacology 1978, 16, 1, doi: 10.1159/000136803; b) R. M. Wenger, T. G. Payne, M. H. Schreier, 'Progress in Clinical Biochemistry and Medicine', Vol 3, 1986, Springer, Berlin, Heidelberg, doi: 10.1007/9783-642-70998-2 5; c) E. K. Schmitt, C. M. Moore, P. Krastel, F. Petersen, Curr. Opin. Chem. Biol. 2011, 15, 497, doi: 10.1016/j.cbpa.2011.05.018.

[3] D. J. Cohen, R. Loertscher, M. F. Rubin, N. L. Tilney, C. B. Carpenter, T. B. Strom, Ann. Intern. Med. 1984, 101, 667, doi: 10.7326/0003-4819-101-5667.
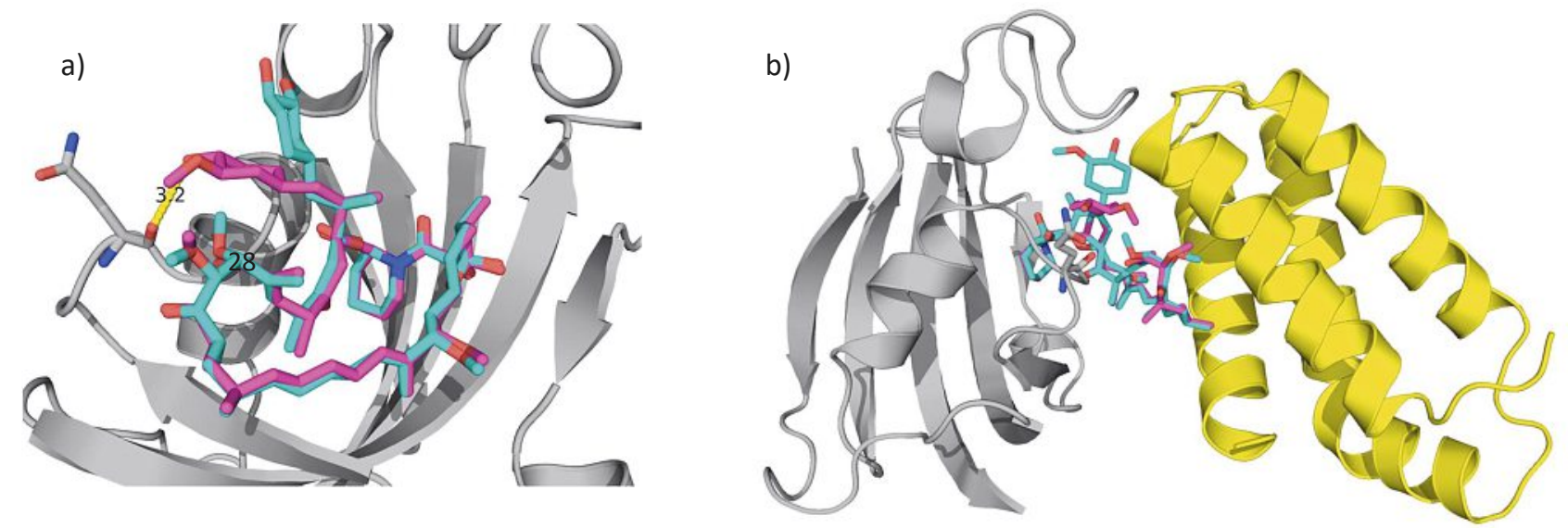

Fig. 10. a) Overlay of rapamycin (magenta) and 28-O-methylrapamycin (21, cyan) bound to FKBP12. b) Overlay of FKBP12-bound 28-O-methylrapamycin (21, cyan) with the FKBP (grey) - rapamycin (magenta) - FRB (yellow) X-ray structure. ${ }^{[23]}$ 
[4] a) C. Vézina, A. Kudelski, S. N. Sehgal, J. Antibiot. 1975, 28, 721, doi: 10.7164/antibiotics.28.721; b) S. N. Sehgal, H. Baker, C. Vézina, J. Antibiot. 1975, 28, 727, doi: 10.7164/antibiotics.28.727.

[5] R. Y. Calne, D. S. J. Collier, S. Lim, S. G. Pollard, A. Samaan, D. J. G. White, Lancet 1989, 334, 227, doi: 10.1016/S0140-6736(89)90417-0.

[6] T. Ochiai, K. Sakamoto, Transplant. Proc. 1988, 20, 209.

[7] F. J. Dumont, M. J. Staruch, S. L. Koprak, M. R. Melino, N. H. Sigal, J. Immunol. 1990, 144, 251 .

[8] W. Cao, P. Mohacsi, R. Shorthouse, R. Pratt, R. E. Morris, Transplantation, 1995, 59, 390

[9] J. Liu, J. D. Farmer Jr, W. S. Lane, J. Friedman, I. Weissman, S. L. Schreiber, Cell, 1991, 66, 807, doi: 10.1016/0092-8674(91)90124-H.

[10] T. Weichhart, in 'mTOR. Methods in Molecular Biology (Methods and Protocols)', Vol 821, Eds T. Weichhart, Springer, 2012, p. 1.

[11] The following assays were used: FKBP12 - the ability of the compounds to bind to FKBP12 in a competitive binding assay was determined. MLR two way murine mixed lymphocyte reaction. IL6-dep.prol. - IL6-stimulated proliferation of an IL6-dependent hybridoma clone. Results were expressed as relative $\mathrm{IC}_{50}=\mathrm{IC}_{50}$ test compound / $\mathrm{IC}_{50}$ reference. The reference in the FKBP12 binding assay was FK506 $\left(\mathrm{rIC}_{50}\right.$ rapamycin $\left.=0.6\right)$ while in the cellular assays it was rapamycin $\left(\mathrm{rIC}_{50}\right.$ rapamycin $\left.=1\right)$. $\mathrm{rCC}_{50}<1$ indicates increased activity compared to the reference while $\mathrm{rIC}_{50}>1$ indicates lower activity. For details see ref. [12].

[12] W. Schuler, R. Sedrani, S. Cottens, B. Häberlin, M. Schulz, H.-J. Schuurman, G. Zenke, H.-G. Zerwes, M. H. Schreier, Transplantation 1997, 64, 36.
[13] a) D. Yohannes, S. J. Danishefsky, Tetrahedron Lett. 1992, 33, 7469, doi: 10.1016/S0040-4039(00)60797-9; b) R. J. Steffan, R. M. Kearney, D. C. Hu, A. A. Failli, J. S. Skotnicki, R. A. Schiksnis, J. F. Mattes, K. W. Chang, C. E. Caufield, Tetrahedron Lett. 1993, 34, 3699, doi: 10.1016/S00404039(00)79204-5.

[14] J. I. Luengo, A. L. Konialian, D. A. Holt, Tetrahedron Lett. 1993, 33, 991, doi: 10.1016/S0040-4039(00)77473-9.

[15] J. I. Luengo, L. W. Rozamus, D. A. Holt, Tetrahedron Lett. 1994, 35, 6469, doi: 10.1016/S0040-4039(00)78248-7.

[16] G. D. Van Duyne, R. F. Standaert, S. L. Schreiber, J. Clardy, J. Am. Chem. Soc. 1991, 113, 7433, doi: 10.1021/ja00019a057.

[17] D. Romo, S. D. Meyer, D. D. Johnson, S. L. Schreiber, J. Am. Chem. Soc. 1993, 115, 7906, doi: 10.1021/ja00070a058.

[18] S. Cottens, R. Sedrani, WO Patent Appl. No. WO964180, 1996.

[19] S. Cottens, R. Sedrani, WO Patent Appl. No. WO9516691, 1995.

[20] a) J. I. Luengo, A. Konialian-Beck, L. W. Rozamus, D. A. Holt, J. Org. Chem. 1994, 59, 6512, doi: 10.1021/jo00101a006; b) J. I. Luengo, A. KonialianBeck, D. A. Holt, Tetrahedron Lett. 1995, 36, 7823, doi: 10.1016/00404039(95)01661-Z.

[21] S. Cottens, R. Sedrani, WO Patent Appl. No. WO9409010, 1994.

[22] R. Sedrani, S. Cottens, J. Kallen, W. Schuler, Transplantation Proc. 1998, $30,2192$.

[23] J. Choi, J. Chen, S. L. Schreiber, J. Clardy, Science, 1996, 273, 239, doi: 10.1126/science. 273.5272 .239

[24] J.A. Kallen, R. Sedrani, S. Cottens, J. Am. Chem. Soc. 1996, 118, 5857, doi: $10.1021 /$ ja954328h 